\title{
Parasite prevalence in a village in Burkina Faso: the contribution of new techniques
}

\author{
Simona Fortunato ${ }^{1}$, Barbara Castagna ${ }^{1}$, Maria Rita Monteleone ${ }^{1}$, Raffaela Pierro ${ }^{1}$, Giuseppe Cringoli ${ }^{2}$, \\ Fabrizio Bruschi ${ }^{1}$ \\ ${ }^{1}$ Department of Translational Research, N.T.M.S., University of Pisa and AOU Pisana, Pisa, Italy \\ ${ }^{2}$ Department of Pathology and Animal Health, University of Napoli Federico II, Napoli, Italy
}

\begin{abstract}
Introduction: Parasites are a major public health problem in developing countries. A coproparasitological and immunoparasitological study was conducted in Burkina Faso, in the rural village of Touguri, in November and December 2011. The coproparasitologic analysis was conducted in the pediatric population and seroprevalence surveys were conducted in the adult population to research intestinal, blood, and helminth parasites.

Methodology: The coproparasitologic study was performed on stool samples using two diagnostic methods - standard microscopy and the FLOTAC technique. The total of 49 stool samples analyzed were obtained from children between two months and eleven years of age. The serology study was carried out to evaluate the prevalence of $P$. falciparum, Echinococcus spp., Tenia solium, and A. lumbricoides using different immunological techniques such as ELISA and Western Blot techniques. The study population included 85 adult patients between 15 and 70 years of age.

Results: Results of coproparasitological analyses showed Hymenolepis nana as the only helminth found, in $28.6 \%$ of the total number of patients. Results of serological evaluation revealed a practically null prevalence of Echinococcus, Taenia solium, and Ascaris lumbricoides, and a $77.64 \%$ prevalence of Plasmodium falciparum.

Conclusions: Despite the small number (especially in terms of coprological samples) of individuals examined, this study showed that the parasite prevalence in a rural area of Burkina Faso has a significant impact in the general population, particularly in children. Another finding was that FLOTAC had a higher sensitivity than the widely used ethyl ether-based concentration technique for coprological sample analysis.
\end{abstract}

Key words: Burkina Faso; Hymenolepis nana; FLOTAC; malaria; Echinococcus spp.; cysticercosis; Ascaris lumbricoides

J Infect Dev Ctries 2014; 8(5):670-675. doi:10.3855/jidc.3660

(Received 15 April 2013 - Accepted 05 August 2013)

Copyright (C) 2014 Fortunato et al. This is an open-access article distributed under the Creative Commons Attribution License, which permits unrestricted use, distribution, and reproduction in any medium, provided the original work is properly cited.

\section{Introduction}

Parasites represent an important cause of mortality and morbidity in the world, especially in developing countries. Hot and humid climates, high population density, very low hygienic conditions, the presence of insect vectors, few economic resources available to invest in prevention programs, and the uses and habits of the populations in developing countries enable parasites to spread more easily $[1,2]$.

With regard to intestinal parasites, it is estimated that two billion people in the world are affected by helminthiasis, caused mainly by Ascaris lumbricoides, Ancylostoma/Necator spp., Hymenolepis nana, and Trichuris trichiura [3]; the most common protozoa are Entamoeba histolytica, which affects about 50 million people, and Giardia duodenalis, which affects approximately 200 million people [4]. The transmission, especially via the fecal-oral route, is favored by the contamination of water and soil due to the absence of adequate sanitation typically found in rural areas. Parasites transmitted by protozoa are undoubtedly the most widespread types. Malaria seems to be responsible for 250-500 million febrile illnesses and more than one million deaths per year worldwide.

For Burkina Faso, a country at position 174a of 177 countries (in 2004) in the Human Development Index of the United Nations Development Programme, parasites are a major public health problem.

With regards to malaria, which plays a major role in the health and economic situation in Africa, most infections occur during or immediately after the short rainy season - between June and October - and 99\% of malaria infections are due to $P$. falciparum. It also seems that Burkina Faso has a prevalence and mortality higher than other West African countries. In 
fact, a study that compared the situation in Burkina Faso with that in Gambia showed that the estimated mortality from malaria is 15.4 per 1,000 per year in Burkina Faso, compared to 9.4 in Gambia, with a rate of annual inoculations per person of 80 and 50, respectively [5].

Another important problem for the country, and more generally for all tropical countries, is represented by zoonoses, in particular cysticercosis, toxoplasmosis, leishmaniosis, and echinococcosis.

Considering intestinal parasites, according to a retrospective study performed between 1997 and 2007 in Burkina Faso of 904,733 patients who visited public health centers distributed throughout the country, as many as $54.7 \%(495,081)$ were positive, $8 \%$ of which infections were due to helminths, $32 \%$ to protozoa, and $14.7 \%$ to other parasites. In addition, the emergence of intestinal parasites is significantly different between desert regions $(38.9 \%$ in the Sahel) and those more humid $(65.8 \%$ in the East) and between rural and urban areas [6].

This study aimed to establish and quantify the prevalence of some parasitic infections in a rural setting in Burkina Faso, in patients who attended the village's consulting room with various symptomatology and to the malnutrition center in particular, by way of coproparasitologic surveys of the pediatric population and through seroprevalence surveys in the adult population. Another aim of this study was to compare the results with other studies previously conducted on African populations and to compare the sensitivity of FLOTAC with the ethyl ether-based concentration technique for the detection of $H$. nana eggs in fixed stool samples.

\section{Methodology}

\section{Coproparasitology}

Stool samples

The study was conducted in the period between 8 November 2011 and 6 December 2011, and involved 56 patients, 7 of whom did not return their stool sample, thus resulting in 49 samples analyzed by the standard coproparassitologic technique and by the FLOTAC technique (Flotac, Napoli, Italy).

Each patient was questioned and examined. Informed consent for collecting samples was requested from each patient. A Para-Pak EcoFix container used to collect stool samples was delivered to the parents of those children who do not have signs or symptoms attributed to intestinal parasites, to investigate the correlation between symptoms and parasitosis.
The total of 49 stool samples were obtained from children between 2 months and 11 years of age in November 2011 from a rural setting in Burkina Faso. The children attended the village's consulting room with various symptomatology and the malnutrition center. Of the 49 children, 26 were female and 23 were male, with a mean age of 3 years for females and 3.5 years for males. Approximately 1-2 grams of feces were preserved in ecological fixative [7], in a ratio of 1 to 3 , at room temperature.

The coproparasitologic study was performed on Burkinabè stool samples using two diagnostic techniques. One was the ethyl ether-based concentration technique using stool samples fixed in Para-Pak Plus EcoFix (Meridian Diagnostics, Milan, Italy). The second one was the FLOTAC basic technique with flotation solution (FS) 7 [8].

\section{Coproparasitological methods}

The ecological-fixed stool samples for helminth were carefully filtered (at least $5 \mathrm{~mL}$ ) and centrifuged at $240 \times \mathrm{g}$ for 10 minutes. The supernatant was poured off and the sediment was mixed with $9 \mathrm{~mL}$ sodium chloride $(\mathrm{NaCl} 0.9 \%)$. After 10 minutes of incubation, the solution was divided into two aliquots of $5 \mathrm{~mL}$, one for ethyl ether concentration, the other one for FLOTAC [8,9]. For the FLOTAC basic technique for $H$. nana diagnosis, the tubes were filled with the bestperforming flotation solution FS7 (zinc sulphate, density $=1.35$ ) [8]. The suspension was transferred into one of the two $5 \mathrm{~mL}$ chambers of a FLOTAC-100 apparatus, centrifuged at $120 \times \mathrm{g}$ for 5 minutes, and translated to separate the floating eggs from the sedimented debris. All eggs visible through the ruled window of the reading disk were enumerated under a microscope at 100x magnification.

\section{Serological analyses}

The serology study was carried out to evaluate the prevalence of $P$. falciparum, Echinococcus spp., Cisticercus spp., and A. lumbricoides in Burkinabè using different immunological techniques such as ELISA and Western Blot techniques.

The study population included 85 adult patients between 15 and 70 years of age who attended the village's consulting room with various symptomatology. There were a total of 46 females $(54.1 \%)$ and 39 males (45.9\%), with a mean age of 34.5 years and 31.9 years, respectively.

After receiving informed consent, blood samples were taken from patients' fingertips and put on absorbing paper that was dried in air and then put into 
a plastic bag which was then firmly closed to prevent contamination.

The blood samples were reconstituted before analysis. This procedure involved putting each absorbent $12 \mathrm{~mm}$ of diameter paper disk in $1.6 \mathrm{~mL}$ of phosphate buffer Tween 20 4\% tot and Bovine Serum Albumin $0,4 \%$ tot solution, in micro-agitation for 90 minutes.

The ELISA kits utilized respectively were Echinococcus IgG (K 7621) for E. granulosus and E. multilocularis (NovaTec, Dietzenbach, Germany), Ascaris lumbricoides IgG (LUCIO - Medical ELISAnal von minden, Regensburg, Germany) and Malaria ELISA (DRG, Marburg, Germany). The Western Blot kits, confirmation test, were Echinococcus - Western Blot IgG (LDBIO Diagnostic, Lyon, France) and Cysticercosis - Western Blot IgG (LDBIO Diagnostic, Lyon, France). Each kit was used as indicated into the manufacturers' instructions.

\section{Results}

Coproparasitological analyses

Of the 49 samples tested, $57.14 \%$ (28 samples) were positive, of which 15 with poliparasitosis (53.57\% of positive cases). Among the 15 cases with poliparasitosis, there were 5 cases with the contemporary presence of helminth and protozoa and 10 cases with different protozoa.

Hymenolepis nana was the only helminth found, in 14 of the 28 positive samples $(28.6 \%$ of the total number).

Giardia duodenalis was found in 12 samples (24.49\%), Entamoeba histolytica/dispar in 7 (14.29\%), Entamoeba coli in 7 (14.29\%), Blastocystis hominis in $3(6.12 \%)$, Entamoeba spp. in $3(6.12 \%)$, Iodamoeba buetschilii in 2 (4.08\%), and Endolimax nana in $1(2.04 \%)$ sample (Tables 1 and 2).

Of the 28 positive samples, 12 patients $(42.85 \%)$ had symptoms correlated with intestinal parasites.

FLOTAC with SF7 allowed for detection of Hymenolepis nana in 9 samples that were previously tested negative by conventional microscopy, increasing the prevalence from $8.1 \%$ to $28.6 \%$. This method also made possible a quantitative estimate of eggs per gram of feces $(\mathrm{EPG}=\mathrm{N} \times 1.2 / 0.25)$, evidencing the presence of low concentrations in positive samples (maximum concentration $=845$ EPG).

\section{Serological analyses}

The study involved a total of 85 patients.

Investigations to find specific antibodies for Echinococcus, Plasmodium spp., Ascaris lumbricoides, and Taenia solium we carried out.

For Echinococcus, the antibody search was carried out first on 42 samples, of which all were negative in the screening test, with index (optical density/cut-off) between 2.3 and 8.85 (negativity $<9$ ). In consideration of these results and the data reported in the literature regarding the prevalence of this parasite in the country, which has extremely low prevalence values in both humans and definitive hosts, the search was not continued in the remaining samples.

For Plasmodium spp., of 85 samples tested, $77.64 \%$ were positive. Of these 66 positive patients, 38 were females $(57.6 \%)$ with a mean age of 35.8 years and range between 15 and 70 years, and 28 were males $(42.4 \%)$ with a mean age of 35 years and range

Table 1. Poliparasitism found in the pediatric population

\begin{tabular}{ccc}
\hline Patients positive for parasite presence in the stool & $\mathbf{2 8 / 4 9}$ & $* * *$ \\
\hline Monoparasitism & $13(46.43 \%)$ & \\
Biparasitism & $9(32.14 \%)$ & $15(53.57 \%)$ \\
Presence of three parasites & $6(21.43 \%)$ & \\
\hline
\end{tabular}

Table 2. Parasite species identified and their prevalence

\begin{tabular}{cc}
\hline Parasite & Occurrence n (\%) \\
\hline Hymenolepis nana & $14(28.6 \%)$ \\
Giardia duodenalis & $12(24.49 \%)$ \\
Entamoeba histolytica/dispar & $7(14.29 \%)$ \\
Entamoeba coli & $7(14.29 \%)$ \\
Blastocystis. hominis & $3(6.12 \%)$ \\
Entamoeba spp. & $3(6.12 \%)$ \\
Iodamoeba buetschilii & $2(4.08 \%)$ \\
Endolimax nana & $1(2.04 \%)$ \\
\hline
\end{tabular}


between 19 and 65 years. In negative samples, 10 were females $(52 \%)$ with a mean age of 33.3 years and range between 20 and 50 years, and 9 were males with a mean age of 37 years and range between 20 and 52 years. The maximum absorbance values were 1.928 , 2.874, and 2.231, respectively.

For Ascaris lumbricoides, the 85 samples analyzed were $100 \%$ negative, with indexes between 0.71 and 4.15 (negativity $<9$ ).

Western blot confirmation, performed on 3 samples for Echinococcus and on 11 for Cysticercus, was negative in $100 \%$ of the samples. For Echinococcus, the 3 samples selected were those which, although they tested negative, had an index closest to the cut-off.

The 11 samples analyzed for cysticercosis were chosen randomly. Of these, 3 belonged to males with a mean age of 36 years and range between 28 and 48 years, and 8 to females with a mean age of 34.4 years and range between 15 and 60 years.

\section{Discussion}

Parasites continue to be a major public health problem in developing countries, where microscopic investigation is still the most widely used diagnostic tool in parasitology. It is therefore essential to have a reliable diagnostic method that is rapid and costeffective for early detection and management of helminths and protozoa.

In Burkina Faso, a country with 15,224,780 inhabitants, $79.8 \%$ of the population lives in rural areas. Our study was conducted in the rural municipal district of Tougourì (76,345 inhabitants) in the province of Namentenga, where $94.9 \%$ of the population lives in rural setting [10]. It is also important to note, in reference to the diffusion of some parasitic disease, that $80 \%$ of the population in Tougourì and in Burkina Faso is Muslim.

Regarding intestinal parasites, a retrospective study of Burkina Faso that included 904,733 patients showed a prevalence of $8 \%$ for helminths. The most frequently occurring helminths were found to be Schistosoma mansoni, Taenia spp., Ascaris lumbricoides, Strongiloides stercoralis, Enterobius vermicularis, and Hymenolepis nana [6]; in our study, however, Hymenolepis nana was the only helminth found in the coproparasitological study. Regarding protozoa, the results in that study are similar to ours.

Regarding epidemiological data in Burkina Faso, $H$. nana was the helminth encountered most frequently - found in $3.99 \%$ of patients - though its incidence has decreased in recent years [11]. In other African countries, the situation is similar. In Mali, a prevalence lower than $1 \%$ has been reported [12]; in Morocco, prevalence ranging from $7.2 \%$, in stool samples from regions using raw wastewater for agriculture to $0.6 \%$ in control populations that do not practice wastewater irrigation has been reported [13]. In Egypt, the prevalence has been reported to range from $6.2 \%$ to $16 \%[14,15]$. In Zimbabwe, where the prevalence was $21 \%$, the infections occurred more frequently in younger children in urban areas but in older children in rural areas. The prevalence in urban areas (24\%) was higher than in rural areas (18\%) [16]. It is also important to note that infection by $H$. nana often remain asymptomatic. Severe infections, supported by more than 20,000 eggs per gram of feces, cause enteritis with or without diarrhea, abdominal pain, and loss of appetite. Children may experience more severe intestinal symptoms, which can include epilepsy, probably due to the toxic action that the products of the inflammation cause. In chronic cases, skin manifestations can appear $[17,18]$.

Diagnosis of $H$. nana and $H$. diminuta relies on the microscopic detection of eggs in fecal samples [19]. Ethyl acetate or ether-based concentration techniques [8] are recommended to increase sensitivity, as egg numbers can be very low. Eggs can also be readily observed in Kato-Katz thick smears [20], a widely used diagnostic method in intestinal helminth surveys $[21,22]$. As an alternative to traditional concentration techniques, the FLOTAC apparatus has been developed $[23,9]$. In human parasitology, this tool has proved to be more sensitive than the ether concentration technique and multiple Kato-Katz thick smears for the diagnosis of soil-transmitted helminths (STHs) [24-26]. It has been reported that FLOTAC had a higher sensitivity than the widely used ethyl ether-based concentration technique [27].

The large discrepancy between the findings for $H$. nana from our study and those reported in another study in Burkina Faso in 2012 (28.6\% and 3.99\%, respectively) [11] can be attributed to the small number of samples we analyzed and to the rural area origin of sample, or to the higher sensitivity of the technique we used.

Furthermore, in the present study, FLOTAC showed a higher sensitivity than the widely used ethyl ether-based concentration technique: prevalence equal to $35.7 \%$ with standard method, in comparison with $57.14 \%$ with FLOTAC. It is important to note that the best flotation solution for $H$. nana diagnosis was zinc sulphate (density $=1.20$ ) [27], which is slightly 
different from the one we used (SF7, zinc sulphate, density $=1.35$ ).

Although the standard microscopy technique is a valid method for the diagnosis of parasitic diseases in developing countries, the FLOTAC technique has proved to be an efficient coprodiagnostic technique for detecting intestinal parasite infections because, besides being multivalent, sensitive, simple technologically, and low cost, allows for not only the accurate detection of poliparasitosis, but also for the evaluation of parasitic burden, a fundamental parameter in the diagnosis and follow-up of helminth infection. Specifically, $H$. nana can be added to the growing list of human intestinal parasites which are reliably diagnosed with the FLOTAC technique. These results should be confirmed by other groups in areas where hymenolepiasis is endemic [27].

Regarding the serological results, the prevalence of Echinococcus was practically null.

This result might be expected, considering a prevalence study conducted by the Ministry of Animal Resources, carried out in Burkina Faso, which found a prevalence of $0.007 \%$ in cattle [28].

On the contrary, other African countries such as those bordering on the Mediterranean (Morocco, Libya, Algeria, and part of Egypt) and, to a lesser extent, those in East Africa (Kenya, Sudan, Ethiopia, Uganda, and Tanzania) are more affected by this parasitic infection [29].

A comparable situation to echinococcosis was observed also for cysticercosis. Even for this parasitic infection, our results showed a practically null prevalence. According to the previously mentioned study carried out in Burkina Faso, cysticercosis was present in only $0.57 \%$ of pigs examined [28]. It should be noted that our study was performed in a rural setting, where the pork is mostly sold rather than eaten, and in a mostly Muslim population that does not consume pork. To our knowledge, data in the literature that indicate the actual prevalence of this parasite in Burkina Faso in humans are lacking.

The search for anti-Plasmodium antibodies showed positive results in $77.64 \%$ of individuals examined. In fact, an estimate of the intensity of transmission of the disease is important to quantify the problem and focus, monitor, and evaluate its control.

In comparison to the other method usually used, the determination of serum positivity is now considered the most appropriate and accurate methodology for estimating the extent of transmission (prevalence) and it is considered the most steady method as well as the one less subjected to seasonal variation [30]. As reported in a study performed in collaboration with local researchers, in Burkina Faso the EIR increases from the dry season to the rainy season from 1 to 10 in urban areas and from 50 to 200 in rural areas [31].

Finally, the seroprevalence for Ascaris lumbricoides was null. This negativity, although unexpected because this nematode is well distributed in African equatorial countries, was confirmed by our coproparasitological study in the pediatric population where the parasite was not found, by the data obtained in the local laboratory (unpublished data), by the retrospective study cited above (which showed a prevalence of $0.5 \%$ ), and by other epidemiological studies [32]. Our results are in agreement with observations from the northern region of Burkina Faso (where the village of Tougourì is situated), where a high prevalence of malaria but low or null prevalence of $A$. lumbricoides was reported [32].

We are aware that our study is limited by a small number of individuals and coprological samples analyzed; however, it has value when the situation in remote rural areas of African countries are considered, from which data are more frequently collected than from urban areas, opening the way to further studies of a larger number of individuals of both pediatric and adult ages who live in this geographical region.

\section{References}

1. Alemu A, Atnafu A, Addis Z, Shiferaw Y, Teklu T, Mathewos B, Birhan W, Gebretsadik S, Gelaw B (2011) Soil transmitted helminths and Schistosoma mansoni infections among school children in zarima town, northwest Ethiopia. BMC Infect Dis 11: 189.

2. Guidetti C, Ricci L, Vecchia L (2011) Eziologia delle parassitosi intestinali in un campione di studenti mozambicani. Le infezioni in medicina 3: 157-165.

3. Matthys B, Bobieva M, Karimova G, Mengliboeva Z, Vreni JR, Hoimnazarova M, Kurbonova M, Lohourignon LK, Utzinger J, Wyss K (2011) Prevalence and risk factors of helminthes and intestinal protozoa infections among children from primary schools in western Tajikistan. Parasit Vectors 4: 195.

4. Minenoa T, Avery MA (2003) Giardiasis: recent progress in chemotherapy and drug development. Curr Pharm Des 9: 841855.

5. Ndugwa RP, Ramroth H, Müller O, Jasseh M, Sié A, Kouyaté B, Greenwood B, Becher H (2008) Comparison of all-cause and malaria-specific mortality from two West African countries with different malaria transmission patterns. Malar J 7: 15 .

6. Cissè M, Coulibaly SO, Guiguemdé RT (2011) Epidemiological features of intestinal parasitic infection in Burkina Faso from 1997 to 2007. Med Trop 71: 257-260. 
7. Pietrzak-Johnston SM, Bishop H, Wahlquist S, Moura H, De Oliveira Da Silva N, Pereira Da Silva S, Nguyen-Dinh P (2000) Evaluation of Commercially Available Preservatives for Laboratory Detection of Helminths and Protozoa in Human Fecal Specimens. J Clin Microbiol 38: 1959-1964.

8. Cringoli G, Rinaldi L, Maurelli MP, Utzinger J (2010) FLOTAC: new multivalent techniques for qualitative and quantitative copromicroscopic diagnosis of parasites in animals and humans. Nat Protoc 5: 503-515.

9. Soren L, Becker SL, Laurent K, Lohourignon LK, Speich B, Rinaldi L, Knopp S, N'Goran EK, Cringoli G, Utzinger J (2011) Comparison of the Flotac-400 Dual Technique and the Formalin-Ether Concentration Technique for Diagnosis of Human Intestinal Protozoon Infection. J Clin Microbiol 49: 2183-2190.

10. Burkina Faso DHS, 2010 Preliminary Report. Enquête Démographique et de Santé (EDS-IV) et à Indicateurs Multiples (MICS). EDSBFMICS IV. BURKINA FASO 2010. Rapport Préliminaire. Ministère de l'Economie et des Finances; Institut National de la Statistique et de la Démographie, Ouagadougou, Burkina Faso. MEASURE DHS, ICF Macro, Calverton, Maryland, USA. Available: http://siteresources.worldbank.org/INTPRH/Resources/37637 4-1303736328719/BurkinaFaso3212web.pdf. Accessed 12 February 2012.

11. Ouermi D, Karou DS, Ouattara I, Gnoula C, Pietra V, Moret R, Pignatelli S, Nikiema JB, Simpore J (2012) Prevalence of intestinal parasites at Saint-Camille medical center in Ouagadougou (Burkina Faso), 1991 to 2010. Med Sante Trop 22: 40-44.

12. Dabo A, Sow MY, Sangaré L, Maiga I, Keita A, Bagayoko Y, Kouriba B, Doumbo O (2003) Transmission of schistosomiasis in an urban population and prevalence of intestinal helminthiasis in Bamako, Mali. Bull Soc Pathol Exot 96: 187-190

13. Habbari K, Tifnouti A, Bitton G, Mandil A (2000) Intestinal parasitosis and environmental pollution: 1343 pediatric cases in Beni-Mellal, Morocco. Tunis Med 78: 109-114.

14. Hassan SI (1994) Parasitic infections in primary and secondary schools in Giza Governorate, Egypt. J Egypt Soc Parasitol 24: 597-601.

15. Khalil HM, el Shimi S, Sarwat MA, Fawzy AF, el Sorougy AO (1991) Recent study of Hymenolepis nana infection in Egyptian children. J Egypt Soc Parasitol 21: 293-300.

16. Mason PR, Patterson BA (1994) Epidemiology of Hymenolepis nana infections in primary school children in urban and rural communities in Zimbabwe. J Parasitol 80: 245-250.

17. King CH, Fairley JK (2010) Cestodes (tapeworms). Hymenolepis nana. In Mandell GL, Bennet JE, Dolin R, editors. Principles and practice of Infectious disease. New York: Churchill Livingstone. 3609-3610

18. Marseglia GL, Marseglia A, Licari A, Castellazzi AM, Ciprandi G (2007) Chronic urticaria caused by Hymenolepis nana in an adopted girl. Allergy 62: 821-822.

19. Schantz PM (1996) Tapeworms (cestodiasis). Gastroenterol Clin N Am 25: 637-653.

20. Katz N, Chaves A, Pellegrino J (1972) A simple device for quantitative stool thick-smear technique in schistosomiasis mansoni. Rev Inst Med Trop São Paulo 14: 397-400.

21. Bergquist R, Johansen MV, Utzinger J (2009) Diagnostic dilemmas in helminthology: what tools to use and when? Trends Parasitol 25: 151-156.
22. Speich B, Knopp S, Mohammed KA, Khamis IS, Rinaldi L, Cringoli G, Rollinson D, Utzinger J (2010) Comparative cost assessment of the Kato-Katz and FLOTAC techniques for soil-transmitted helminth diagnosis in epidemiological surveys. Parasit Vectors 3: 71

23. Cringoli G (2006) FLOTAC, a novel apparatus for a multivalent faecal egg count technique. Parassitologia 48: 381-384.

24. Utzinger J., Rinaldi L, Lohourignon LK, Rohner F, Zimmermann MB, Tschannen AB, N'goran EK, Cringoli G (2008) FLOTAC: a new sensitive technique for the diagnosis of hookworm infectious in humans. Trans R Soc Trop Med Hyg 102: 84-90.

25. Knopp S, Glinz D, Rinaldi L, Mohammed KA, N'Goran EK, Stothard JR, Marti H, Cringoli G, Rollinson D, Utzinger J (2009) FLOTAC: a promising technique for detecting helminth eggs in human faeces. Trans R Soc Trop Med Hyg 103: 1190-1194

26. Knopp S, Rinaldi L, Khamis IS, Stothard JR, Rollinston D, Maurelli MP, Steinmann P, Marti H, Cringoli G, Utzinger J (2009b) A single FLOTAC is more sensitive than triplicate Kato-Katz for the diagnosis of low-intensity soil transmitted helminth infections. Trans R Soc Trop Med Hyg 103: $347-$ 354.

27. Steinmann P, Cringoli G, Bruschi F, Matthys B, Lohourignon LK, Castagna B, Maurelli MP, Morgoglione ME, Utzinger J, Rinaldi L (2012) FLOTAC for the diagnosis of Hymenolepis spp. infection: proof-of-concept and comparing diagnostic accuracy with other methods. Parasitol Res 111: 749-754.

28. Coulibaly ND, Yameogo KR (2000) Prevalence and control of zoonotic diseases: collaboration between public health workers and veterinarians in Burkina Faso. Acta Trop 76: $53-$ 57.

29. Grosso G, Gruttadauria S, Biondi A, Marventano S, Mistretta A (2012) Worldwide epidemiology of liver hydatidosis including the Mediterranean area. World J Gastroenterol 18: 1425-1437.

30. Corran P, Coleman P, Riley E, Drakeley C (2007) Serology: a robust indicator of malaria transmission intensity? Trends Parasitol 23: 575-582.

31. Modiano D, Sirima BS, Sawadogo A, Sanou I, Parè J, Konatè A, Pagnoni F (1998) Severe Malaria in Burkina Faso: Influence of age and Trasmission level on Clinical Presentation. Am J Trop Med Hyg 59: 539-542.

32. Mwangi TW, Bethony JM, Brooker S (2006) Malaria and helminth interactions in humans: an epidemiological viewpoint. Ann Trop Med \& Parasitol 100: 551-570.

\section{Corresponding author}

Fabrizio Bruschi

Department of Translational Research, N.T.M.S.

University of Pisa Medical School

Via Roma, 5556126 Pisa, Italy

Phone: +39(050) 2218547

Fax: +39(050) 2218557

Email: fabrizio.bruschi@med.unipi.it

Conflict of interests: No conflict of interests is declared. 